\title{
Molecular Beam Epitaxy of Materials Interfaces with Atomic Precision
}

\author{
(C) Klaus H. Ploog
}

Paul Drude Institute for Solid State Electronics,

101117 Berlin, Germany

E-mail: KlausH.Ploog@t-online.de

In this contribution a few selected examples to engineer material interfaces in nanostructured solids with atomic precision by means of molecular beam epitaxy (MBE) are presented. The examples include $2 D$ electron gas systems for quantum transport and mesoscopic physics, quantum cascade lasers, Sb-based materials, ferromagnet-semiconductor heterostructures, as well as oxide materials for electronics and quantum physics. Finally, the prospects to fabricate novel van-der-Waals heterostructures are briefly discussed. 